\title{
ASO Visual Abstract: The Value of Paratracheal Lymphadenectomy in Esophagectomy for Adenocarcinoma of the Esophagus or Gastroesophageal Junction: a Systematic Review of the Literature
}

\author{
Amaia Gantxegi, $\mathrm{MD}^{1}$, B. Feike Kingma, $\mathrm{MD}$, $\mathrm{PhD}^{2}$, Jelle P. Ruurda ${ }^{2}$, Grard A. P. Nieuwenhuijzen, Md, $\mathrm{PhD}^{3}$, \\ Misha D. P. Luyer, $\mathbf{M d}, \mathbf{P h D}^{3}$, and Richard van Hillegersberg ${ }^{2}$ \\ ${ }^{1}$ Vall d'Hebron Hospital Universitari, Barcelona, Spain; ${ }^{2}$ Department of Surgery, University Medical Center Utrecht, P.O. \\ Box 85500, Utrecht, The Netherlands; ${ }^{3}$ Catharina Hospital Eindhoven, Eindhoven, The Netherlands
}

The optimal extent of mediastinal lymphadenectomy for distal esophageal and gastroesophageal junction (GEJ) adenocarcinomas is a matter of debate, especially regarding paratracheal lymphadenectomy. This review (https:// doi.org/10.1245/s10434-021-10810-8) aimed to provide objective evidence for the reported incidence of lymph node metastasis in the upper mediastinum.

(C) Society of Surgical Oncology 2021

Published Online: 30 October 2021

R. van Hillegersberg

e-mail: R.vanHillegersberg@umcutrecht.nl
Supplementary Information The online version contains supplementary material available at https://doi.org/10.1245/s10434021-10895-1.

DISCLOSURE Jelle Ruurda and Richard van Hillegersberg are proctors for Intuitive Surgical Inc. The remaining authors have no conflicts of interest.

Publisher's Note Springer Nature remains neutral with regard to jurisdictional claims in published maps and institutional affiliations. 